\title{
P 119 CAUSES OF SLEEP DISTURBANCE IN A SPECIALIST PALLIATIVE CARE UNIT
}

Jayne Glynn, Sue Gale, Shila Tank. E\&N Herts NHS Trust, Middlesex, England

10.1136/bmjspcare-2014-000654.160

Background Sleep disturbance is a common and distressing problem in patients who are terminally ill and may intensify symptoms such as pain, depression, and/or anxiety. Staff in a Specialist Palliative Care inpatient unit felt that they were inadvertently and partly responsible for unnecessarily disturbing patients during the night, however, the frequency and precise nature of what caused patients to wake was unknown. A survey was undertaken to identify causes of disturbance and explore ways of reducing them.

Methods Data were collected on all direct patient contact between $10 \mathrm{pm}$ and $6 \mathrm{am}$. Data including time of disturbance, mode of seeking attention, and the primary reason for nurse assistance were recorded along with secondary data relating to additional needs and outcomes.

Results 301 disturbances occurred in 27 nights. 86\% (60/70) of inpatients had at least one need (range 1-4). Most needs were between 22:01-24:00 hrs (29\%) followed by 00:01-02:00 hrs $(25 \%)$ and 02:01-04:00 hrs (23\%). Patients used their call bell $(58 \%)$, called out $(18 \%)$ or were observed by a healthcare professional. Primary reason for seeking help related to elimination (31\%), symptomatic (26\%), wanted food/drink (13\%), repositioning $(10 \%)$, confused/talking in their sleep $(6 \%)$, others $(15 \%)$. Of the 10 symptoms reported pain was the most common followed by breathlessness, insomnia, cough/hiccups. Patients frequently articulated additional needs once their primary need had been met, for example, wanted analgesia, then asked for hot drink, assistance to the toilet, fan, etc. These subsequent needs resulted in 500 activities being carried out. Nurses and/or their activities were not reported as a cause for waking patients, however, when meeting an individual's needs other patients opportunistically expressed help/needs.

Conclusion Nursing activities do not appear to disturb patients. Ways of reducing nocturia in this client group are limited, however, more could be done to explore symptom management, such as prophylactic medication use. Pre-bedtime snacks should be provided and encouraged. 